\title{
Utilizing RFID Technology in Chinese Power Grid Logistics Management
}

\author{
Rui Liu ${ }^{1, a^{*}, \text { Shiyuan Lin }}{ }^{2, b}$ Bryan Hubbard ${ }^{3, c}$ \\ 1,2 School of Economy and Management, North China Electric Power University, \\ Changping District, Beijing,102206, China \\ ${ }^{3}$ Department of Building Construction Management, Purdue University, \\ West Lafayette, Indiana, 47906, USA \\ a liurui@ncepu.edu.cn, blinshiyuan2009@163.coml, 'bhubbard@purdue.edu
}

\begin{abstract}
Keywords: RFID technology; smart grid; power grid logistic feature; present management status; application methods; technology development obstacles

Abstract. Radio Frequency Identification (RFID) is an advanced technology, which can be applied to automatic data identification, acquisition and distribution. When utilized in power grid logistics management, this technology will assist to improve the logistics management efficiency. However, RFID hasn't been used widely so far and only a few people realized it in power grid Co. Meanwhile about application methods, related researches haven't been classified by power grid logistics feature at present, which would result in readers' misunderstanding. Also the literatures haven't been combined with new management idea, like supply chain management, to build a comparatively complete application system. Accordingly, this paper will file the present documents firstly and specifically introduce what is RFID? Why should we use RFID in power grid logistics management? And how to use this technology? Secondly this paper will explain the obstacles to RFID employment and related solutions. Last but not least, this paper will line out the defects in present researches and propose RFID future development directions, which may provide some references to researchers to some extent.
\end{abstract}

\section{Background}

Power Grid is the vital base of economic and social development. Thus Realizing power grid running safely and steadily is the significant guarantee to build a well-off society in an all-round way and to construct a harmonious socialistic society [1].In May, 2009, the State Grid Co. proposed an overall development target, which is the construction of strong smart grid. The 'Smart' based on grasping information from complete grid promptly and effectively. And this base is on the premise of massive amounts of information acquisition terminal and data distribution and conversion [7].

RFID, as an advanced information-acquisition technology, can be applied for automatic data identification, acquisition and distribution. This technology has been employed in various industries and companies logistics management and realized management efficiency boost and cooperated relationship improvement among upstream-downstream firms. Nevertheless, there are a few researches about utilizing RFID in power grid and even less in grid logistics.

Hence, this paper will file the present documents firstly and specifically introduce what is RFID; Why we should use RFID in power grid logistics management; And how to use this technology according to grid logistics feature. Also this paper will explain the obstacles to RFID deployment and corresponding solutions. Meanwhile this paper will propose RFID future development directions, which may supply the reference to readers to some extent.

\section{What is RFID}

Radio Frequency Identification (RFID), also known as E-tag, is an automatic non-line-of-sight data identification technology, which obtains information through radio-frequency signal and interlink with management system. When it works, the reader send special radio-frequency to wake up tags in a 
certain scope. Then awaken tags send self-information to reader through tag antenna. After obtaining information, reader will modem and transfer that to the management system. This technology can be used to identify dynamic objects and scan piles of tags at the same time. Also the whole working procedure needs no human participation. RFID technology consists of tag, reader, antenna and management system.

According to related literature [10-11], the advantages of RFID are shown as follows.

(1) Durability. Most grid logistics are exposed to the outdoors and will be affected by weather conditions. Tags of RFID can continue working flawlessly in harsh conditions $\left(-200-40^{\circ} \mathrm{C}\right)$, adapting to grid logistic management demands comparing with barcodes used at present.

(2) Non-line-of-sight. RFID technology can realize non-line-of-sight and pile scan within a certain working scope, which won't be influenced by objects status (dynamic or static). This feature decrease the waiting time when check-in or check-out logistics, simplifies logistic-check work, increases the handling capacity of warehouse, and improves the logistics management efficiency.

(3) Accuracy \& Instantaneity. Recording data through RFID will reduce the mistakes from human during logistics management and improve the data-record accuracy. Meanwhile this technology achieves the logistics throughout tracking and sharing. Moreover RFID guarantees the information distribution promptly and avoids the repetitive work about data.

(4) Automation. It is estimated that RFID could yield labor savings of up to $36 \%$ in order piking and a $90 \%$ reduction in verification costs. It plays an important role in management costs savings and efficiency improvements.

\section{What is Grid Logistics Management}

In accordance with logistics features, they can be divided into two parts, namely Operation and Maintenance logistics (O\&M) and Construction logistics.

(1) O\&M Logistics. These logistics are used for power grid device technological-remoulding, operation, and maintenance. The management procedure is the groups submit logistics demands to management departments, which they belong to, for approval. After approval the grid Co. will organize the procurement all together. Then the logistics will be stored in the warehouses and when be in needed, they will be sent to the demand spot [4]. Every group in the power grid company should predict O\&M logistics demand beforehand according to the last year's information and device operating condition. The procedure is shown in figure 1.

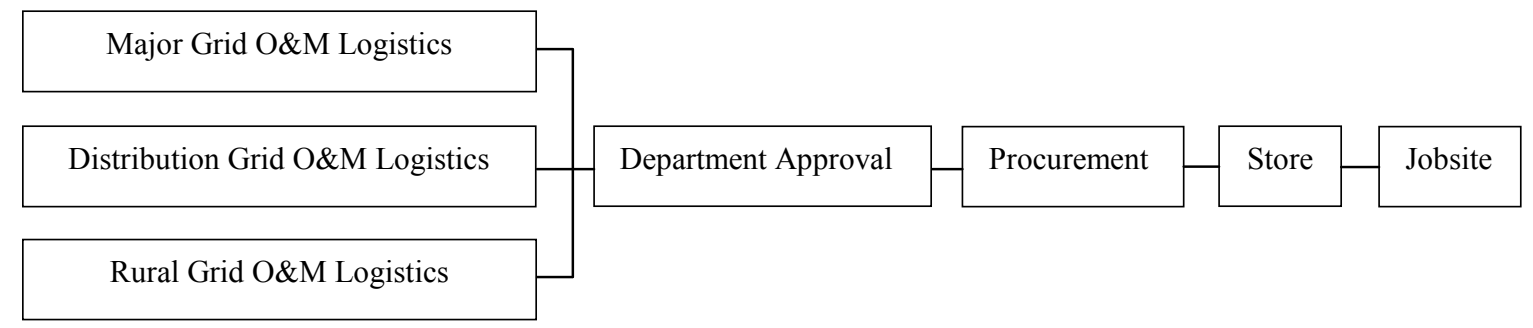

Fig.1.The procedure of O\&M logistics management

(2) Construction Logistics. These logistics are the equipment and material for power grid construction, which is with the one-off feature. The departments in the power grid company are in charge of making self-projects setting. After the project approval, they will predict related logistics demands. And then one department will summarize all demands and call for bid invitation. It is worth noting that the logistics will be delivered to construction jobsite directly by manufacture instead of being stored in the warehouses [4]. The procedure is shown as below in Figure 2. 


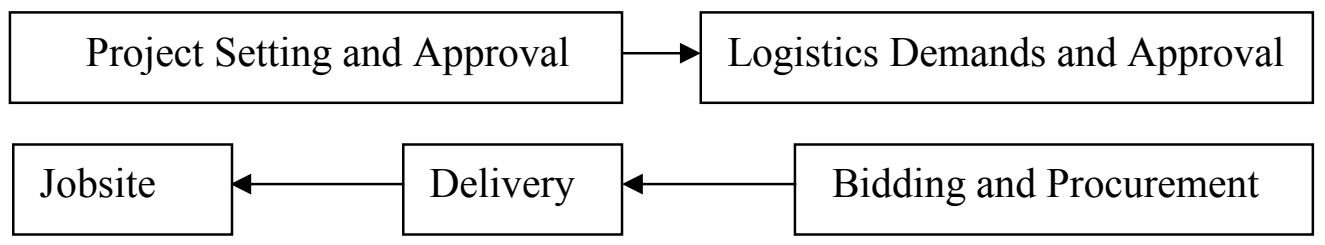

Fig.2. The procedure of construction logistic management

\section{Why should use RFID in Power Grid Logistics Management?}

Electric Power is one of the pillar industries of national economy. With rapidly economic development, the electric power logistics have boosted from type, quantity and value, which means the management should involve the whole life-cycle instead of stock tracking only[13].

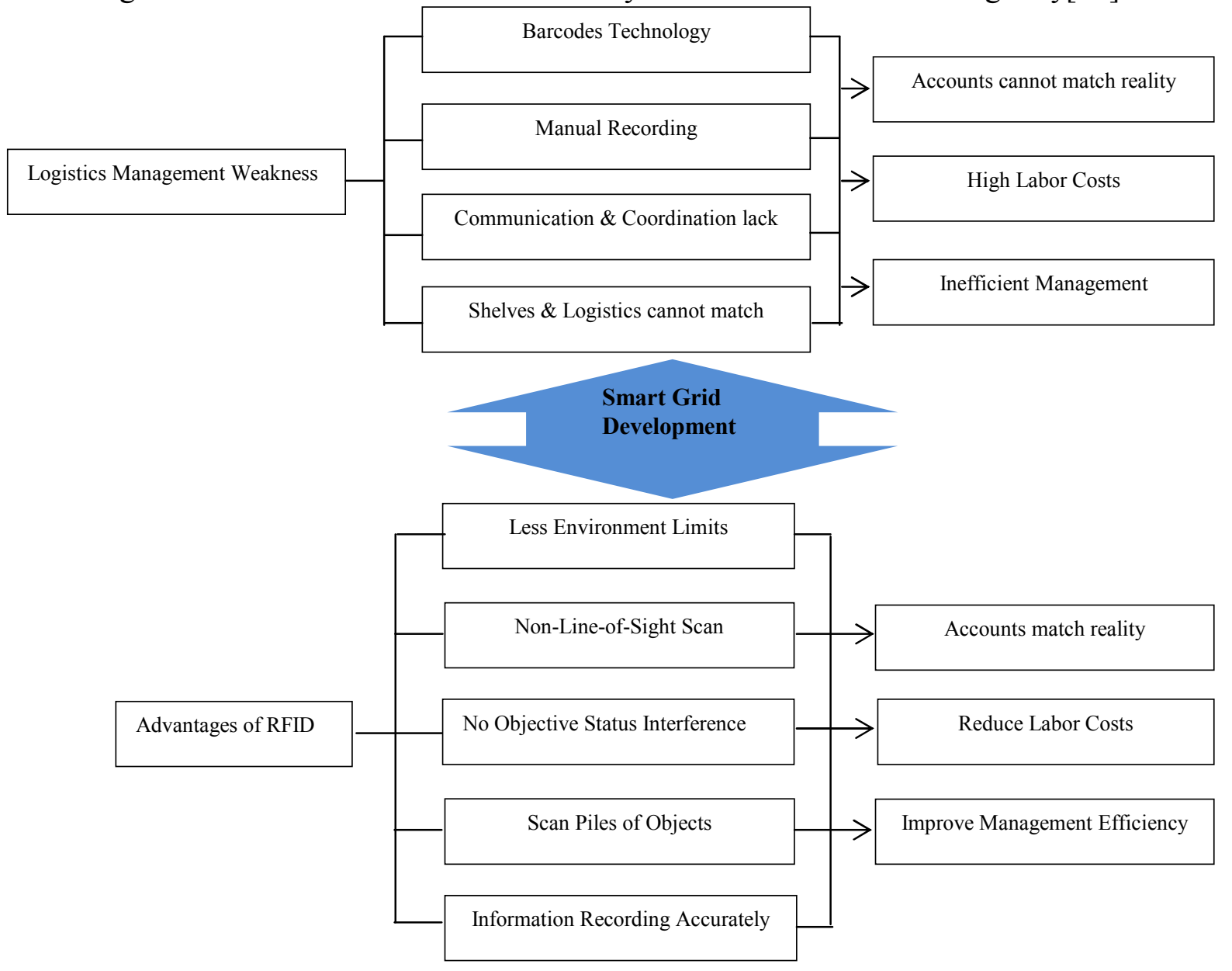

Fig. 3. Summary Map of Meanings of RFID Deployment

\section{RFID meets power grid logistics management requirements.}

In May, 2009, the State Grid Co. proposed an overall development target, which is the construction of strong smart grid. The Smart Grid is characterized by informatization, digitization, automation and interaction [1]. The 'Smart' of smart grid based on grasping information from complete grid promptly and effectively. And this base is on the premise of massive amounts of information acquisition terminal and data distribution and conversion. Therefore information acquisition technology is an indispensible part of smart grid [5].

Massive information collection asks for proper data acquisition device. RFID is an advanced data acquisition technology with large identification scope, better anti-interference ability, less environmental limits, smaller size and diverse forms, meeting almost all the requirements from power grid. Applying RFID technology into Smart Grid can realize electric power capital information automatic acquisition, check, identification, management, and inspection. 


\section{RFID can improve power grid logistics management status at present.}

(1) Information Acquisition. In power grid Co., barcodes are used in information collection. But this technology requires line-of-sight when reading, which will reduce the working efficiency. Meanwhile the paper characteristics material is not suitable in outdoor environment. On the contrary, RFID tags comparably durable and can fit the harsh conditions. Also the RFID tags realize the non-line-of-sight scan and won't be interference by objects status (dynamic or static).

(2) Information Recording. When recording information, power grid Co. still employs the traditional manual input and modify. This method is inefficient, inaccurate and the data could not be updated in time. Whereas with RFID, the automatic identification can reduce the mistakes during logistics check-in and check-out.

(3) Information distribution. In power grid Co., the capital is managed by different departments in different phases. But for lacking of communication and coordination, the management phases comparatively independent and could not realize the whole life-cycle management [7]. However RFID technology makes the information full-process tracking and sharing come true, and avoids the data repetitive operation as well.

(4) Management System. The warehouse of power grid Co. is still under the older traditional management method, which could not match the shelves with logistics. When check-in, check, and check-out, the only way is relying on the warehouse keepers' experience. Such an outdated method seriously hinders the management efficiency [14]. While RFID technology, with management system assistance, the warehouse can achieve the shelves and logistics matching accurately.

Accordingly RFID technology can realize accurate matching between account and reality, reduce manual mistakes, decrease labor costs, and improve power grid management status.

\section{How to Utilize RFID in Power Grid Logistics Management}

\section{Apply RFID to O\&M logistics management}

The key for RFID application is in the warehouse management. According to the related literature [14-15], the applied methods are concluded as follow.

(1) Logistics Procurement. After initialize setting from power grid Co., the RFID tags will be supplied to manufactures. The manufacturers would be requested to input information about logistics model, quantity, production batches and destination departments. Before delivering to the power grid warehouse, the manufacturers should send these information to warehouse management system to ease the system preprocessing and shelves allocation.

(2) Shelves Management. The warehouse keepers will stick RFID tags on the shelves. Through readers the stock allocation serial number will be input into the tags and after scanning this information will be recorded in the management system. When logistics shelving and un-shelving, the tags data should be changed.

(3) Logistics Check-in. RFID reader will be installed on the warehouse's door so as to scan tags directly and send data to the management system directly. After data confirmed, system will allocate shelves number from preprocessing program and send related information to readers. Then readers will input information into tags separately. The warehouse keepers shelve logistics according to the system arrangement and confirm that in the system to ensure information integrity and accuracy.

(4) Stock Management. The information in the system will be update according to the realistic condition. When the stock quantity under the necessary volume, lost or shelves mismatching happen, the system will warn the person in charge..

(5) Logistics Check. According to the managers' requests, the management system will set and send checking command to the terminal. The warehouse keepers check and scan RFID tags on the required shelves according to the system's message. After information uploaded, the system will generate summary sheets to classify logistics have been checked and avoid repetitive work.

(6) Logistics Check-out. After receiving stock requisition from related departments, warehouse keepers will take out the logistics according to system arrangement and upload related information. 
When freight vans go through the entry the readers will scan the tags again to make sure the data is in line with the system record.

The completed procedure is shown in figure 4.

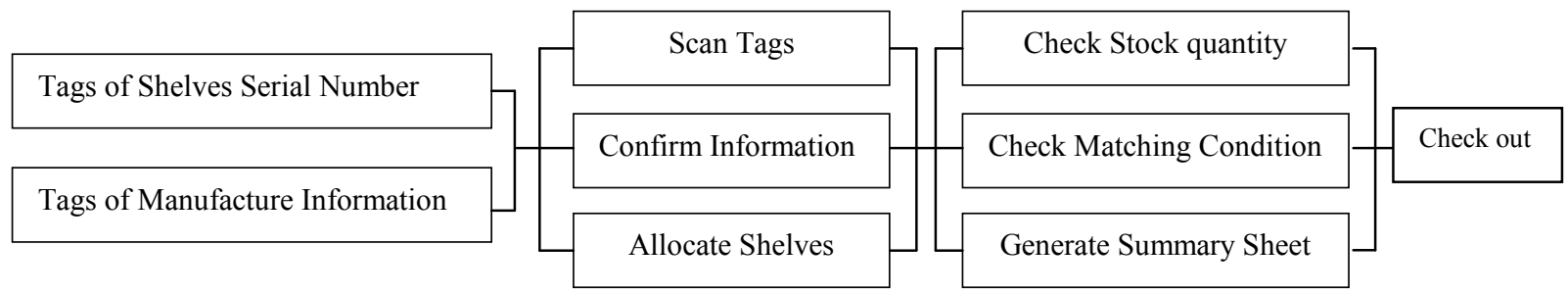

Fig.4. The Procedure of O\&M Logistics Management

\section{Apply RFID to Construction Logistics Management}

The key for RFID application is on the jobsite management. According to the related literature [16-19], the applied procedure is concluded as follows.

(1) Logistic Procurement. Like the phase in O\&M, after signing contacts and initialize setting from power grid Co., the related tags information will be input by the manufacturers. Also the management system will preprocess before the logistics arriving.

(2) Enter Jobsite. Before construction, the account information should be downloaded to the reader. When logistics arrived, the readers will scan the tags and confirm logistics according downloaded information. After confirmation, the logistics will be classified stocked.

(3) Jobsite Management. The keepers will stick different kind of RFID tags to equipment, device and tools according to their features. during using, system will acquire and chasing their spot and details, which eases the management procedure, distinguishes different owners and avoids the tools lost. Meanwhile like O\&M logistics, readers will warn when the quantity under necessary volume.

(4) Installation Supervise. Sticking RFID tags to the expensive equipment and letting the operators to manage them in different phases, will separate the responsibility, ease the system record, and supervise the installation clearly and efficiently.

(5) Visitor Control. The RFID tags will record main information of jobsites labor. When people want to enter the jobsite, they have to take the RFID tags with proper authority. This method will limit the people without authority, protect the construction jobsites as well as ensure the jobsite safe.

Accordingly, the RFID technology will ease the logistics, equipment, device, tools and visitors control, guarantee the installation supervise and clear the managers' responsibilities.

\section{Obstacles of RFID Application}

Tags Cost. The costs of RFID tags will be 8 times more than the barcodes. Thus, in the practical application, we can combine the RFID technology with barcodes. According to the IOR ratio, we will apply RFID tags to the logistics with high output, but barcodes to the other one. However with the continuous development of RFID, the cost of it will decrease step by step and be accepted by more and more clients [8].

Information Transfer. With the RFID technology development, the RFID tags will replace barcodes gradually. But if we research and develop a brand new logistics management system based on RFID technology, and abandon the older barcodes one, will waste a lot of time and capital. Hence we can transfer the information between RFID and barcodes [12].

Standard Unified.There are three main kinds of RFID codes standards at present, namely ISO/IEC system from international organization for standardization, EPC system from International Article Numbering Association (EAN) and UID system from Japan [12]. Not unified standards result in Non-conformant codes, which affect communication protocol. Therefore power grid Co. should pay attention to standardization problem, standardize manufacture and utilization of RFID products, and stimulate the speedy and healthy development of RFID technology.

Reliability Problem. The open interconnection design of RFID is the basic reason for reliability problem. In practical application, RFID technology confronts 5 main risks, namely overheard, deceive, 
reject, track and clone [20-22]. Corresponding protection strategies are divided into cryptographic algorithm and asymmetric algorithm. Although with elasticity, the cryptographic algorithm cannot keep away all the risks; and the resistance ability of asymmetric algorithm is limited more than the former one. But with the technology development, the reliability of RFID will improve gradually and finally possess standard and practical defense function [22].

Management Thought.The power grid Co. as a large-scale state-owned $\mathrm{Co}$, has little connection with up-stream manufactures. in facts, the risks of logistics demand change are taken by manufacturers and the management thoughts of power grid Co. haven't transformed into the competing-market one. Thus, the company should change their management thoughts, enhance the RFID publicity, and cultivate related labor to utilize this new technology [25].

\section{Conclusion}

In recent years, the power grid corporation has developed the Smart Grid construction, applied whole life-cycle theory into management, and enhanced ERP system. In order to innovate and improve power grid logistic operation mode, the corporation gradually admitted and utilized the RFID technology.

In such a background, this paper filed related research results and combined with two main power grid logistics features, introduce the RFID technology from what is it; why should we use it in the power grid logistics management; and how to employ this technology. Meanwhile, this paper explains the present status of RFID application obstacles namely costs, transfer, standardization, reliability, and management thoughts.

Accordingly there are lack of literature illustrates RFID according to the power grid logistics features. And most research based on horizontal life-cycle theory but seldom consider the vertical supply chain management thoughts. Also there are inner and outer supply chains in power grid Co. the inner one, among departments is more completed than the outer one cause the cooperation lack between the corporation and up-stream manufactures. all of these aspects could become the breakthrough of future researches.

\section{References}

[1] Strong Smart Grid research group, "Independent innovation, international leading strong smart grid comprehensive study report”, (2009).

[2] Ying Rui, "The power grid logistics supplier choice under supply chain environment", Wuhan University of Technology, Wuhan, (2009).

[3] Wei Benyou, "the power grid logistics supplier cooperation and application based on supply chain", Wuhan University of Technology, Wuhan, (2009).

[4] Li Sha, "The research about power grid logistics base on supply chain management", Huazhong University of Science and Technology, Wuhan, (2010).

[5] Chen Jinghui, Wu Fei Chen Yameng, Huang Xiaoyun, Wang Hongwei, Xie Shimulin, "The research about power grid equipment management system application", Technology Information, vol 9, (2014), pp: 210-211.

[6] An Jianrong. " Utilizing RFID technology in power grid logistics management", City Construction Theory (Electronic Edition), vol. 32, (2012).

[7] Zong Jingsong, “The research about RFID application in smart grid data acquisition”, North China Electric Power University, Beijing, (2012).

[8] Luo Feng, "The application about RFID in supply chain logistics management", Southwest Jiaotong University, (2009). 
[9] Lung-Chuang Wang, Yu-Cheng Lin, Pao H. Lin. "Dynamic mobile RFID-based supply chain control and management system in construction". Advanced Engineering Informatics, vol 21 (2007), pp: 377-390.

[10] Katina Michael, Luke McCathie. "The Pros and Cons of RFID in Supply Chain Management”. Proceedings of the International Conference on Mobile Business, ICMB (2015).

[11] Duan Qichang, Guo Tingting, Huang Xiaogang, "The RFID application in power grid logistics management system”, Chinese Journal of Scientific Instrument, vol 30, (2009), pp:135-138.

[12] Lu Kun, "The RFID coding plan in power grid capital”, Information and communication network technology council annual conference, No. T21, (2011).

[13] Tan Yi, "the system design about power grid assets management based on RFID technology", journal of the guangxi academyof sciences, wol 20, no.1, (2012). Pp: 80-82.

[14] Liu Li, “The RFID application in power grid logistics delivery center", Market Weekly, vol 6, (2012), pp:111-112.

[15] Hong Songchen, “Apply RFID technology in power grid logistics stock and delivery”, Chinese \& Foreign Entrepreneurs, vol 5, (2013), pp: 135-137.

[16] Lu Shuang, Hong Fanghua, Zhu Lijun, “ Apply RFID technology in power grid logistics whole process management”, East China Electric Power, vol 40, (2012).

[17] Qin Li, “The power grid equipment whole life-cycle management based on RFID technology", South Power Grid Technology, vol 3, (2014), pp: 119-123.

[18] Xiao Junye, “The prospect of RFID technology in power grid construction", Engineer and Technology, vol 5, (2011), pp: 463-465.

[19] Hu Jie, Zong Ping, "The research about RFID reliability strategy target to internet", Computer Technology and Development, vol 21, no. 5, (2011), pp: 151-154.

[20] Yang Haidong, Yang Chun, "The research about RFID reliability problems", Microcomputer Information, vol 24, no.8, (2008), pp: 238-240.

[21] Colle P, Jakobsson M., Juels A., SyversonP..UniversalReencryption for Mixnets. In Okamoto, T. (Ed), RSA Conference Cryptographers Track. (2004), pp: 163-178.

[22] Sheng Xianfei, Wu Guowei, Zhou Hui, Zhu Weimin, "The discussion about appling RFID technology in power grid whole life-cycle management", Internet of Things, vol 12, (2012), pp: 28-31.

[23] Tan Yi, "The System design about power grid assets management based on RFID technology", journal of the guangxi academyof science, vol 28, no.1, (2012), pp: 80-82.

[24] Li Xing, "The research about applying RFID and GIS in smart distribution grid inspection system", North China Electric Power University, Beijing, (2013).

[25] Luo Chunbing, Peng Yan, Luo Bing, "Review of RFID technology development and application”, Communication Technology, vol12, (2009). 